\title{
Mobile diagnostics in outbreak response, not only for Ebola: a blueprint for a modular and robust field laboratory
}

\author{
R Wölfel ${ }^{12}$, K Stoecker $^{12}$, E Fleischmann ${ }^{12}$, B Gramsamer ${ }^{12}$, M Wagner $^{12}$, P Molkenthin ${ }^{12}$, A Di Caro ${ }^{3}$, S Günther $^{42}, \mathrm{~S}$ \\ Ibrahim ${ }^{15}$, GH Genzel $^{1}$, AJ Ozin-Hofsäss ${ }^{6}$, P Formenty ${ }^{7}$, L Zöller ${ }^{1}$ \\ 1. Bundeswehr Institute of Microbiology, Munich, Germany \\ 2. European Mobile Lab Consortium \\ 3. Lazzaro Spallanzani National Institute for Infectious Diseases, Rome, Italy \\ 4. Bernhard Nocht Institute for Tropical Medicine, Hamburg, Germany \\ 5. Current affiliation: US Army Research Development and Engineering Command, Edgewood Chemical Biological Center, \\ Aberdeen Proving Ground, MD, United States \\ 6. European Centre for Disease Prevention and Control, Stockholm, Sweden \\ 7. World Health Organization, Geneva, Switzerland
}

Correspondence: Roman Wölfel (romanwoelfel@instmikrobiobw.de)

Citation style for this article:

Wölfel R, Stoecker K, Fleischmann E, Gramsamer B, Wagner M, Molkenthin P, Di Caro A, Günther S, Ibrahim S, Genzel GH, Ozin-Hofsäss AJ, Formenty P, Zöller L. Mobile diagnostics in outbreak response, not only for Ebola: a blueprint for a modular and robust field laboratory. Euro Surveill. 2015;20(44):pii=30055. D0I:

http://dx.doi.org/10.2807/1560-7917.ES.2015.20.44.30055

Article submitted on 31 May 2015 / accepted on 23 September 2015 / published on 05 November 2015

We established a modular, rapidly deployable laboratory system that provides diagnostic support in resource-limited, remote areas. Developed as a quick response asset to unusual outbreaks of infectious diseases worldwide, several of these laboratories have been used as part of the World Health Organization response to the Ebola virus outbreaks by teams of the 'European Mobile Lab' project in West Africa since March 2014. Within three days from deployment, the first European mobile laboratory became operational at the Ebola Treatment Unit (ETU) in Guéckédou, southern Guinea. Deployment in close proximity to the ETU decreased the turnaround time to an average of $4 \mathrm{~h}$ instead of several days in many cases. Between March 2014 and May 2015, more than 5,800 samples were tested in this field laboratory. Further EMLab units were deployed to Nigeria, Liberia and Sierra Leone in the following months of the Ebola outbreak. The technical concept of the EMLab units served as a blueprint for other mobile Ebola laboratories which have been set up in Mali, Côte d'Ivoire, Sierra Leone and other countries in West Africa. Here, we describe design, capabilities and utility of this deployable laboratory system for use in response to disease outbreaks, epidemiological surveillance and patient management.

\section{Background}

Since December 2013, West Africa has been facing the deadliest outbreak of Ebola virus disease (EVD), previously known as Ebola haemorrhagic fever. The current epidemic has killed within a year of its onset more than 10,300 people as reported from eight affected countries Liberia, Guinea, Sierra Leone, Mali, Nigeria, Spain, the United Kingdom and the United States [1].
It was as early as March 2014 that two European laboratories identified the causative agent as the Zaire ebolavirus [2], which was immediately followed by a response of the World Health Organisation (WHO) with the support of its Global Outbreak Alert and Response Network (GOARN).

EVD usually begins with fever, chills, and general malaise. These and other symptoms, including fatigue, headache, vomiting, diarrhoea, anorexia, and myalgia [3], can also result from a number of other infectious diseases endemic in Africa, e.g. malaria, dengue, leptospirosis and typhus [4]. Therefore, accurate identification of the causative agents is critical for effective containment of the outbreaks and provision of appropriate supportive care to the patients [5-9]. In preparedness for rapid, global response to naturally occurring and emerging infectious disease outbreaks, the International Cooperation and Development Office of the European Commission (DG DevCo) launched the European Mobile field laboratory (EMLab, www.emlab. eu) initiative in 2012. Three mobile laboratory units were established in home bases in Europe (Munich, Germany), West Africa (Nigeria) and East Africa (Tanzania).

The concept of a modular, rapidly deployable field laboratory was previously developed at the Bundeswehr Institute of Microbiology in 2008. The primary goal of the Bundeswehr Medical Mobile Laboratory (BML) initiative was to establish field-deployable diagnostic capabilities for medical surveillance and investigation of unusual disease outbreaks in theatres of military operation. The driving design criterion was to enable 


\section{FIGURE 1}

Mobile Field Laboratory equipment in different phases of deployment

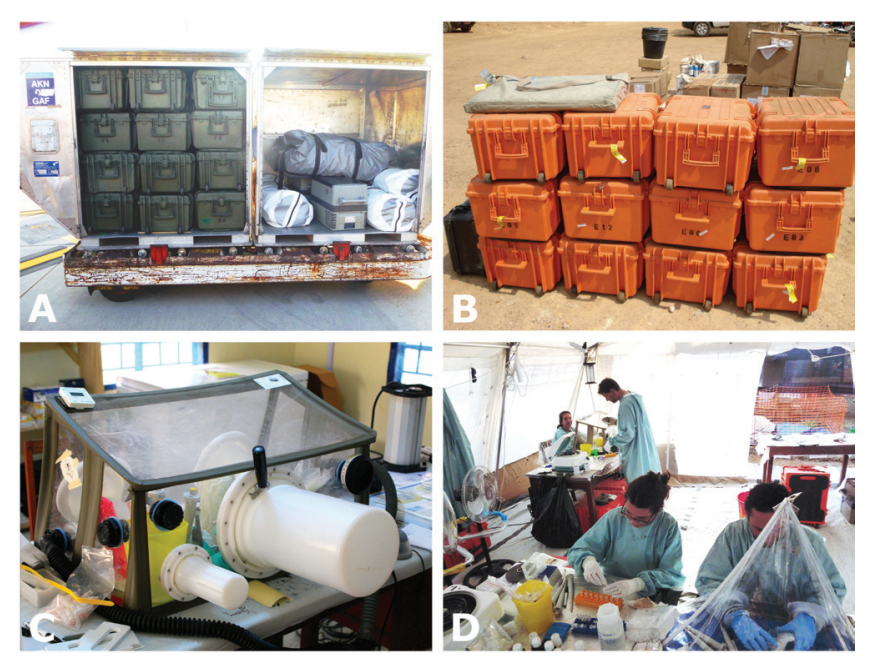

A. Airlift of a complete BML as passenger luggage, including two rapidly inflatable tent systems.

B. EMLab consortium upon arrival in Guéckédou, Guinea in March 2014 .

C. Improved glovebox unit for sample inactivation.

D. First EMLab team providing Ebola diagnostics in a tent inside the MSF treatment centre in Guéckédou, Guinea, April 2014

worldwide deployment within 72 hours with minimal logistical burden (Figure 1). Therefore, modular units with multiple, flexible configurations to accommodate different mission requirements were envisioned. The BML is staffed with a four-person team equipped with the capability to provide confirmatory identification of a variety of bacterial and viral pathogens and biological toxins [10]. From 2008 to 2014 , the BML has successfully been deployed in several missions to the Balkans, South and Central Asia, West Africa, North America and Europe (data not shown). Based on the BML model, the EMLab units were adopted for civilian multinational outbreak response missions in Africa and Europe.

On 26 March 2014, an EMLab team was deployed to Guinea at the request of the Guinean Ministry of Health and WHO GOARN. Within three days, the first mobile laboratory became operational at the Ebola Treatment Unit (ETU) in Guéckédou, southern Guinea, and its impact was evident as the turnaround time from specimen collection to reporting of test result was reduced from days to hours. Between March 2014 and May 2015, more than 5,800 samples were tested in this field laboratory (Table 1). Further EMLab units were deployed to Nigeria, Liberia and Sierra Leone in the following months of the Ebola outbreak. In addition, the technical concept of the EMLab units served as a blueprint for other mobile Ebola laboratories which have been set up in Mali, Côte d'Ivoire, Sierra Leone and other countries in West Africa.

\section{Key elements of mobile laboratory units}

Four elements were identified as essential for effective mobile laboratories: (i) personnel and training, (ii) biosafety and biosecurity management, (iii) methods and equipment and (iv) logistical support. These elements can be adapted to suite specific missions according to the nature of outbreaks, geographic areas, season and climatic conditions, and on-site resources.

\section{Personnel and training}

The human factor is the most critical element for a successful mobile field laboratory mission. Modern laboratory equipment and sophisticated diagnostic methods have to be handled by highly skilled laboratory personnel in the environment of a field laboratory. Previous experience in biosafety level (BSL) 3 or BSL-4 laboratory work can facilitate, but cannot replace, the adaption of the laboratory personnel to the biosafety and biosecurity procedures of a field laboratory. We consider a four-person team as the ideal size for fieldwork over a period of three to six weeks to allow for rest and rotation during peak operations. Handover from one team to the next should consider an overlap of at least two days to ensure seamless transition and continuation of the operation.

Before deployment, all team members should be trained under realistic field conditions. Based on the experience of several laboratory deployments, we developed a training curriculum consisting of 25 modules. It covers realistic scientific, medical, technical and operational challenges that could be encountered in a field situation. A shortened version of this training enables participants to conduct Ebola diagnostics in one of the already established EMLab units in West Africa during the latest outbreak (Table 2).

\section{Biosafety and biosecurity management}

The difficulty of operating and relocating BSL-3 or BSL-4 laboratories in the field environment is well understood [11]. Several container-based or truck-mounted laboratory units have been purported to meet most BSL-3 regulatory requirements. However, those have rarely been deployed worldwide due to their large footprint, heavy weight and high logistical burden.

These constraints often interfere with rapid and timely response because sophisticated air, maritime and land transport infrastructure are needed as well as significant and continuous technical maintenance. Most of these requirements cannot be met in rural areas of developing countries, such as Guinea, Sierra Leone or Liberia.

In general, biosafety in a mobile laboratory is achieved by optimising laboratory practices, equipment safety and qualification of the laboratory staff [12-14]. For example, neither cultivation nor enrichment of 
Schematic setup of the European Mobile Field Laboratory equipment and layout of two independent electrical supply lines in a tent or a fixed building

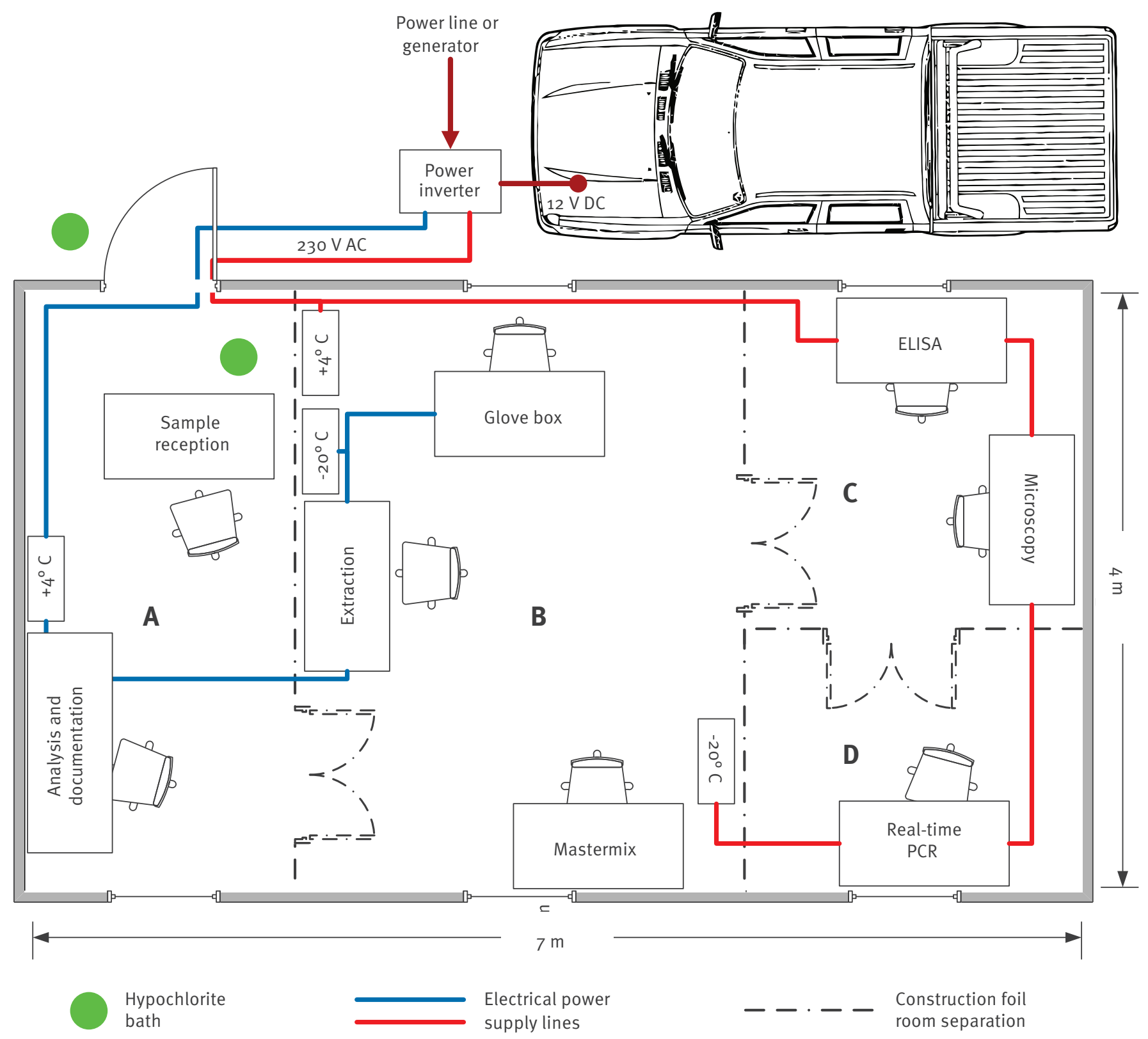

Hypochlorite baths in front of the field laboratory and at the sample reception are used to disinfect the packaging of submitted samples before further processing in the laboratory.

infectious material are foreseen in the mobile laboratory as this would dramatically increase biosafety risks as well as biosecurity risks [15]. The diagnostic procedures used, e.g. qPCR or RT-qPCR, immunoassays or light microscopy, do not depend on living organisms, and the specimens can easily be inactivated by validated chemical or heat treatment methods before further analysis [16-18].

For the protection of laboratory personnel during sample inactivation, two options can be employed: One option is wearing personal protective equipment (PPE), e.g. coverall suits, goggles and FFP-3 face masks within a designated 'hot zone' [6]. However, this option has a number of disadvantages: (i) the time laboratory personnel can work in full protective gear is very limited especially in tropical climate, (ii) a designated separate 'hot zone' is needed, adding another factor to the already complex task of setting up a field laboratory, and (iii) this 'hot zone' has to be considered as contaminated until it has been completely disinfected. The second option is the use of a hermetically sealed glovebox system. Gloveboxes are regarded as the best way to minimise the risk of exposure for workers especially 
TABLE 1

European Mobile Laboratory mission statistics, as per 31 October 2015

\begin{tabular}{|c|c|c|c|c|c|c|c|}
\hline $\begin{array}{l}\text { EMLab } \\
\text { (country) }\end{array}$ & $\begin{array}{l}\text { EMLab } \\
\text { (town) }\end{array}$ & $\begin{array}{l}\text { First sample } \\
\text { tested }\end{array}$ & $\begin{array}{l}\text { Last sample } \\
\text { tested }\end{array}$ & $\begin{array}{l}\text { Operation time } \\
\text { (weeks) }\end{array}$ & $\begin{array}{l}\text { Number of } \\
\text { teams }\end{array}$ & $\begin{array}{c}\text { Samples } \\
\text { tested }\end{array}$ & EBOV-positive (qPCR) \\
\hline Guinea & Guéckédou & 30 Mar 2014 & 3 Apr 2015 & 53 & 15 & 5,842 & 2,231 \\
\hline Guinea & Coyah & 17 Feb 2015 & Ongoing & 30 & 9 & 5,292 & 487 \\
\hline Nigeria & $\begin{array}{l}\text { Enugu and } \\
\text { Port Harcourt }\end{array}$ & 20 Aug 2014 & 1 Oct 2014 & 5 & 2 & 30 & 3 \\
\hline Liberia & Foya & 13 Sep 2014 & 4 Dec 2014 & 12 & 4 & 315 & 81 \\
\hline Sierra Leone & $\begin{array}{l}\text { Freetown } \\
\text { and Kambia }\end{array}$ & 14 Dec 2014 & Ongoing & 46 & 9 & 4,220 & 287 \\
\hline Sierra Leone & Hastings & 22 Dec 2014 & 5 Sep 2015 & 43 & 10 & 4,012 & 343 \\
\hline \multicolumn{5}{|l|}{ Total } & 49 & 19,711 & 3,432 \\
\hline
\end{tabular}

EBOV: Ebola virus; EMLab: European ‘Mobile field laboratory’.

in hot climate. At the same time, heat stress is reduced for the operator since the glovebox renders the PPE dispensable. Only in case of failure of the glovebox, the laboratories teams would rely on PPE (coveralls and powered air-purifying respirators with hoods) for sample inactivation. In addition, the PPE is to be used to safely unpack samples that are too large for the glovebox or in the unlikely event of contamination inside the mobile laboratory environment.

Several flexible film isolator models have previously been used as gloveboxes in field laboratory missions $[5,19]$. Although designated as portable, we found that those units are either too large and heavy to be easily deployed, or, because non-solid walls are used, only minimal negative pressure can be applied to the interior of these gloveboxes. Negative pressure of more than -1 mbar ( -2 mbar is the minimum requirement for stationary BSL-3 glovebox systems [20]) would lead to the collapse of the flexible film walls.

We bridged the gap between biosafety needs and mobility by developing a foldable glovebox system with solid polycarbonate walls (Figure 1C). Its design was based on the basic technical requirements for microbiological safety cabinets described in European Standard EN 12469 [20]. With a negative internal pressure up to -2.5 mbar that can be monitored and a leak rate less than $10 \%$ per $30 \mathrm{~min}$ at an overpressure of 5 mbar, this glovebox combines the safe working environment of a stationary glovebox with minimised packing size. Uncomplicated and safe handling was the main objective for the design of this foldable glovebox system. In the course of the Ebola missions in West Africa, the glovebox design was further optimised for durability and resistance in hot climate. The gloves can be adapted to the individual hand size of the operator to allow comfortable and safe performance of the inactivation protocols. Damaged gloves or sleeves can easily be replaced aseptically during glovebox operation. Two rapid transfer ports of different size, a well-established docking system in the pharmaceutical industry, allow contamination-free, safe and easy transfer of samples, supplies and waste into and out of the glovebox without breaking the containment.

All patient samples are stored in secondary packaging (plastic tubes or bags labelled with bleach resistant markers), floating in buckets filled with hypochlorite until further processing. This ensures thorough surface decontamination. For protection of the laboratory staff, all patient samples are finally unpacked inside the glovebox only. Two field laboratory team members operate the foldable glovebox: One operator is conducting all processing steps in the glovebox while another team member crosschecks and documents all steps with biosafety or diagnostic relevance on the respective laboratory request form. To prevent cross-contamination, samples are inactivated one after another, not simultaneously.

\section{Methods and equipment}

The BML system offers a range of diagnostic technologies, including qPCR, ELISA, immunofluorescence assays (IFA), immunochromatographic tests and microscopy. It is important to emphasise that all assays have to be adapted to and validated on the laboratory equipment before being included in the diagnostic portfolio of the mobile laboratory. Over the past seven years, we established more than 50 assays for 33 different pathogens and toxins that can be used under field conditions with the BML system. Performance of all PCR assays is controlled by the use of either competitive or non-competitive internal controls (IC), as well as extraction, negative and positive controls. Generally, IC are added to lysis buffer and patient serum samples before nucleic acid extraction in order to validate the complete diagnostic process.

For the ongoing Ebola outbreak in West Africa, we decided to focus on the detection of Ebola virus in different body fluids using RealStar Filovirus Screen RT-qPCR Kit 1.0 (Altona Diagnostics, Hamburg, Germany) and of Malaria parasites in the blood of 
TABLE 2

Modular $(71 \mathrm{~h})$ training modules for preparation of mobile laboratory teams

\begin{tabular}{|c|c|}
\hline Introduction in the mobile field laboratory concept & $1 \mathrm{~h}$ \\
\hline Field laboratory setup & $3 \mathrm{~h}$ \\
\hline $\begin{array}{l}\text { Technical instruction on handling of mobile laboratory } \\
\text { equipment }\end{array}$ & $2 \mathrm{~h}$ \\
\hline Immunofluorescence and light microscopy protocols & $2 \mathrm{~h}$ \\
\hline Glovebox maintenance & $1 \mathrm{~h}$ \\
\hline SmartCycler maintenance and repair & $1 \mathrm{~h}$ \\
\hline Personnel protective equipment & $2 \mathrm{~h}$ \\
\hline Power generator operation and maintenance & $1 \mathrm{~h}$ \\
\hline Power converter operation & $1 \mathrm{~h}$ \\
\hline Scenario-based laboratory team training & $24 \mathrm{~h}$ \\
\hline Additional scenario-based team training & $8 \mathrm{~h}$ \\
\hline Sample reception and documentation & $1 \mathrm{~h}$ \\
\hline Documentation, statistics and reporting of data & $2 \mathrm{~h}$ \\
\hline qPCR results interpretation and troubleshooting & $2 \mathrm{~h}$ \\
\hline Gel electrophoresis & $2 \mathrm{~h}$ \\
\hline Storage of samples & $1 \mathrm{~h}$ \\
\hline Preparation of samples for IATA shipment & $2 \mathrm{~h}$ \\
\hline Satellite phone communication & $1 \mathrm{~h}$ \\
\hline VHF radio communication & $2 \mathrm{~h}$ \\
\hline Map and GPS training & $2 \mathrm{~h}$ \\
\hline $\begin{array}{l}\text { Team safety and security in the field, including mission } \\
\text { specific sociocultural aspects }\end{array}$ & $2 \mathrm{~h}$ \\
\hline Advanced laboratory troubleshooting & $2 \mathrm{~h}$ \\
\hline Disinfection regime and waste management & $2 \mathrm{~h}$ \\
\hline Additional laboratory waste management in the field & $1 \mathrm{~h}$ \\
\hline Field laboratory dismantling and packaging & $3 \mathrm{~h}$ \\
\hline
\end{tabular}

GPS: global positioning system; IATA: International Air Transport Association; VHF: very high frequency.

Training modules printed in bold are included in the condensed (40 h) training designed to handle demand for the Ebola outbreak support mission in 2014 and 2015.

the patients (BinaxNow, Alere, Cologne, Germany). The Filovirus RT-qPCR kit includes an IC that allows to check for RT-PCR inhibition. For a valid negative result, the $I C$ and the positive control must show a signal at the correct cycle threshold $(\mathrm{Ct})$ value, and the extraction control must not show amplification. Each run was validated independently by two team members. During the EMLab missions in West Africa, ca $10 \%$ of all runs did not meet these criteria, mainly due to IC failure, and had to be repeated. Additional tests were performed (at the request of the sender) for Ebola virus-negative cases, including West African Lassavirus-specific qPCR, Dengue immunochromatographic tests, Ebola IgG IFA and Malaria microscopy. In the course of the Ebola outbreak in West Africa, EMLab teams have successfully participated in two independent laboratory quality control trials conducted by WHO and the European Network for Diagnostics of Imported Viral Diseases (ENIVD).
Turnaround time is an important indicator of mobile laboratory performance, and can be influenced by many factors, including sample collection, transportation, preparation, analysis, result interpretation and reporting. Time for transportation of samples to a laboratory is the factor contributing most to the total turnaround time. Deployment of the first EMLab unit in close proximity to the ETU in Guéckédou decreased the turnaround time to an average of $4 \mathrm{~h}$ instead of several days in many cases. A typical $12 \mathrm{~h}$ workload for a fourperson laboratory team was 40 samples. The gloveboxbased sample inactivation may present a bottleneck. However, well-trained laboratory teams should be able to process up to 90 samples within 12 hours as demonstrated by several EMLab teams during the peak of the Ebola outbreak in Guinea.

The molecular diagnostics capability of the EMLabs is primarily based on a ruggedised and robust qPCR instrument (SmartCycler, Cepheid, Sunnyvale, CA) with real-time PCR modules than can be programmed independently, which has previously been used in several field laboratory missions $[5,6,21,22]$. Each instrument is able to test up to 16 different samples in parallel using different thermocycling conditions, and a new run can always be started while other runs are still in progress. In contrast to other qPCR instruments, the optical system has no moving parts or bulbs that could be damaged during transport. In case of technical failure of one of the 16 PCR modules the remaining modules are still operational. Furthermore, laboratory team members can easily replace the defective modules in the field.

Given the fact that in remote regions, replacement of broken or lost equipment can be difficult or impossible within a reasonable time frame, a concept of fallback options for essential parts of the field laboratory has been implemented: Key components such as centrifuges, power sources and IT equipment are safeguarded against failure by duplication. If the qPCR cycler would be irrevocably damaged, small conventional thermal cyclers and gel electrophoresis systems are available.

The infrastructural requirements for setting up the mobile laboratory are minimal. Generally, almost every local building can be used as long as it provides at least $28 \mathrm{~m}^{2}$ of space. In several overseas deployments, the rapidly inflatable $B M L$ tent system with prefabricated interior room separations (ADS 1611 B-LABORATORY, OWR, Elztal-Rittersbach, Germany) was successfully used even in remote areas. Without the need for a dedicated 'hot room' for sample inactivation, the mobile laboratory requires a minimum of two rooms, to separate the pre- and post-PCR area (Figure $2 \mathrm{~A} / \mathrm{B}$ and C/D). However, subdivision of the laboratory into four rooms (Figure $2 \mathrm{~A}-\mathrm{D}$ ) is preferred and can be prepared by the team using construction foil, foil doors and duct tape. The complete set-up of the field laboratory takes 4-5 h to operational readiness. The laboratory design adopts 
TABLE 3 A

Complete list of components of the European Mobile Field Laboratory

\section{Module/items}

Biosafety protective equipment 3 packages, $72 \mathrm{~kg}$

Rapid Containment Kit Ver 03, OWR Rittersbach

Laboratory personal protective equipment 2 packages, $36 \mathrm{~kg}$

Single-use protective suit, class 3 b

Hood, 3 M Versaflo S-333G

Blower unit, Micronel $\mathrm{C}_{420}$

Cooling vest, TST Sweden

Laboratory safety googles

Nitrile gloves, long sleeves, large

Nitrile examination gloves, medium

$\mathrm{P}_{3}$ RD filter canister

Pen

Sample preparation 3 packages, $47 \mathrm{~kg}$

Centrifuge, Hettich EBA 21

Centrifugation tube, polycarbonate, $50 \mathrm{ml}$

Paper wipes, pack

Pipette, $100 \mu \mathrm{L}$

Pipette, $1,000 \mu \mathrm{L}$

Plastic bottle $1 \mathrm{~L}$, scaled

Polyethylene construction sheeting, $20 \mathrm{~m}^{2}$

Ring adaptor for gloves

Tape

Tube, cone-shaped, $50 \mathrm{~mL}$

Warning sign 'biohazard'

Waste bags, autoclavable

Pre-PCR 2 packages, $57 \mathrm{~kg}$

Centrifuge, Hettich EBA 21

Centrifuge, VWR Galaxy mini

Mastermix glovebox, Captair Pyramide

QIAamp DNA blood and tissue mini kit

QIAamp stool kit

QIAamp viral RNA kit

Thermomixer

Vortex mixer

Parafilm, pack

SmartCycler PCR tubes

Pen, alcohol resistant

Phosphate-buffered saline tablets, pack

Pipette, $10 \mu \mathrm{L}$

Pipette, $100 \mu \mathrm{L}$

Pipette, $1,000 \mu \mathrm{L}$

Polyethylene construction sheeting, $20 \mathrm{~m}^{2}$

Syringe, $10 \mathrm{~mL}$

Syringe filter, $0.2 \mu \mathrm{m}$

Real-time PCR 2 packages, $60 \mathrm{~kg}$

Real-time PCR, SmartCycler II

PCR termal cycler, Finnzyme Piko

Parafilm, pack

Pen, alcohol resistant

Pipette, $10 \mu \mathrm{L}$

Pipette, $100 \mu \mathrm{L}$

Pipette, $1,000 \mu \mathrm{L}$

Polyethylene construction sheeting, $20 \mathrm{~m}^{2}$

Reaction tube

Tube rack, $0.2 \mathrm{~mL}$

Tube rack, $1.5 \mathrm{~mL}$

Vortex mixer

Waste bags, autoclavable

\begin{tabular}{|c|c|c|} 
& 10 & \\
& 6 & \\
& 4 & \\
& 4 & \\
& 6 & \\
& 20 & \\
\hline & 20 & \\
& 12 & \\
& 5 &
\end{tabular}

Rapid Containment Kit, accessories, set 'Munich'

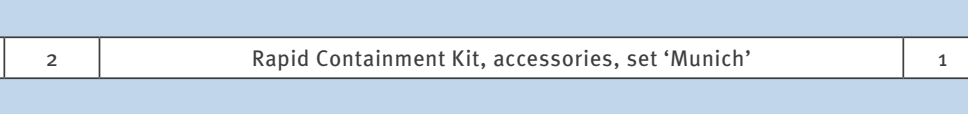

Hose for blower unit

Adapter plug

Adhesive tape

Apron, single-use

Battery charger for blower unit

Rechargeable batteries for blower unit

Rubber boots

FFP-3 respirator mask, single-use

Butyl gloves

\begin{tabular}{|c|c|}
\hline 4 \\
\hline 1 \\
\hline 1 \\
\hline 15 \\
\hline 1 \\
\hline 6 \\
\hline 5 \\
\hline 15 \\
\hline 6 \\
\hline
\end{tabular}

\begin{tabular}{|c|c|}
\hline 1 \\
\hline 12 \\
\hline 10 \\
\hline 1 \\
\hline 1 \\
\hline 3 \\
\hline 1 \\
\hline 2 \\
\hline 1 \\
\hline 20 \\
\hline 6 \\
\hline 20 \\
\hline
\end{tabular}

Bio bottle, class 6.2 packaging Scissors, single-use

Gaiter, pack

Disposable plastic sharps container

Forceps, single-use

Dengue rapid test

HIV 0.5 rapid test

Influenza $A$ and $B$ rapid test

Malaria rapid test

Nitrile examination gloves, large

Nitrile examination gloves, medium

Nitrile examination gloves, small

6

\begin{tabular}{|l|l|l|}
\hline & Nitile examination gloves, small \\
\hline
\end{tabular}

\begin{tabular}{|c|c|c|}
\hline 1 & Adhesive towel drape & 10 \\
\hline 1 & Autoclave tape & 1 \\
\hline 2 & Reaction tube, $2 \mathrm{~mL}$ & 200 \\
\hline 1 & Cooling block & 2 \\
\hline 1 & Filter tips, $10 \mu \mathrm{L}$, pack & 4 \\
\hline 1 & Filter tips, $100 \mu \mathrm{L}$, pack & 4 \\
\hline 1 & Filter tips, $1,000 \mu \mathrm{L}$, pack & 5 \\
\hline 1 & Laboratory coat, single-use & 6 \\
\hline 1 & Laboratory timer & 1 \\
\hline 500 & Micro test tube, $1.5 \mathrm{~mL}$ & 200 \\
\hline 2 & Micro test tube, $1.5 \mathrm{~mL}$ sterile & 100 \\
\hline 10 & Paracord, $20 \mathrm{~m}$ & 1 \\
\hline 2 & Tube rack, $0.2 \mathrm{~mL}$ & 1 \\
\hline 3 & Tube rack, $1.5 \mathrm{~mL}$ & 4 \\
\hline 2 & Tube rack, $50 \mathrm{~mL}$ & 1 \\
\hline 1 & Tube, cone-shaped, $50 \mathrm{~mL}$ & 25 \\
\hline 50 & Storage box & 5 \\
\hline \multicolumn{3}{|l|}{50} \\
\hline 1 & iCore modules for SmartCycler & 3 \\
\hline 1 & Autoclave tape & 1 \\
\hline 1 & Centrifuge, small & 1 \\
\hline 10 & Disposable plastic sharps container & 2 \\
\hline 1 & Filter tips, $10 \mu \mathrm{L}$, pack & 4 \\
\hline 1 & Filter tips, $100 \mu \mathrm{L}$, pack & 4 \\
\hline 1 & Filter tips, $1,000 \mu \mathrm{L}$, pack & 4 \\
\hline 1 & Gaiter, pack & 2 \\
\hline 200 & Laboratory notebook & 2 \\
\hline 1 & Laboratory coat, single-use & 6 \\
\hline 2 & Nitrile examination gloves, large & 70 \\
\hline 1 & Nitrile examination gloves, medium & 30 \\
\hline 25 & Nitrile examination gloves, small & 40 \\
\hline
\end{tabular}


TABLE 3 B

Complete list of components of the European Mobile Field Laboratory

\begin{tabular}{|c|c|c|c|}
\hline \multicolumn{4}{|l|}{ Module/items } \\
\hline Gel electrophoresis, Lonza FlashGel & 1 & Laboratory timer & 1 \\
\hline FlashGel cassette $2.2 \%$ & 9 & Microtitre plate & 10 \\
\hline Electrophoresis power supply, $300 \mathrm{~V}$ & 1 & Pen, alcohol resistant & 2 \\
\hline Nitrile examination gloves, large & 70 & Pipette, $10 \mu \mathrm{L}$ & 1 \\
\hline Nitrile examination gloves, medium & 30 & Pipette, $100 \mu \mathrm{L}$ & 1 \\
\hline Nitrile examination gloves, small & 40 & Pipette, $1,000 \mu \mathrm{L}$ & 1 \\
\hline Adhesive towel drape & 4 & Plastic bottle $1 \mathrm{~L}$, scaled & 2 \\
\hline Autoclave tape & 2 & Polyethylene construction sheeting, $20 \mathrm{~m}^{2}$ & 1 \\
\hline Disposable plastic sharps container & 1 & Purified water, infusion bag, $500 \mathrm{~mL}$ & 4 \\
\hline Filter tips, $10 \mu \mathrm{L}$, pack & 3 & Tube rack, $0.2 \mathrm{~mL}$ & 1 \\
\hline Filter tips, $100 \mu \mathrm{L}$, pack & 3 & Tube rack, $1.5 \mathrm{~mL}$ & 2 \\
\hline Filter tips, $1,000 \mu \mathrm{L}$, pack & 4 & Vortex mixer & 1 \\
\hline Gaiter, pack & 2 & Waste bags, autoclavable & 20 \\
\hline Laboratory coat, single-use & 6 & Micro test tube, $1.5 \mathrm{~mL}$, sterile & 200 \\
\hline \multicolumn{4}{|l|}{ ELISA 1 package, $24 \mathrm{~kg}$} \\
\hline ELISA reader, TECAN Sunrise & 1 & Adhesive seals, pack & 1 \\
\hline Mini-incubator $\left(37^{\circ} \mathrm{C}\right)$, HumaTemp & 1 & Combitip dispenser & 2 \\
\hline Multi-channel pipette $100 \mu \mathrm{L}$ & 1 & Combitips, $10 \mathrm{~mL}$ & 30 \\
\hline Multi-channel pipette $300 \mu \mathrm{L}$ & 1 & Combitips, $1 \mathrm{~mL}$ & 40 \\
\hline Paper wipes, pack & 10 & Microtitre plate & 10 \\
\hline Phosphate-buffered saline tablets & 100 & Filter tips, $100 \mu \mathrm{L}$, pack & 5 \\
\hline Pipette, $100 \mu \mathrm{L}$ & 1 & Graduated pipette, single-use & 10 \\
\hline Plastic bottle $1 \mathrm{~L}$, scaled & 2 & Laboratory timer & 1 \\
\hline \multicolumn{4}{|l|}{ Microscopy 1 package, $31 \mathrm{~kg}$} \\
\hline Microscope, Partec CyScope & 1 & Cover glass & 100 \\
\hline Polyethylene construction sheeting, $20 \mathrm{~m}^{2}$ & 1 & Disposable plastic sharps container & 1 \\
\hline Purified water, infusion bag, $500 \mathrm{~mL}$ & 3 & Glass slides & 100 \\
\hline Staining solution, Gram & 1 & Immersion oil, pack & 1 \\
\hline Staining solution, McFadyean & 1 & Inoculating loop, pack & 3 \\
\hline Storage box for microscope slides & 2 & Laboratory coat, single-use & 8 \\
\hline Tube rack, $50 \mathrm{~mL}$ & 2 & Laboratory timer & 1 \\
\hline Tube, cone-shaped, $50 \mathrm{~mL}$ & 2 & Pipette Pasteur, single-use & 60 \\
\hline Tweezers, single-use & 2 & Nitrile examination gloves, large & 30 \\
\hline Urine beaker with seal & 2 & Nitrile examination gloves, medium & 20 \\
\hline Waste bags, autoclavable & 5 & Paper wipes, pack & 10 \\
\hline Waste bottle $1 \mathrm{~L}$, teflon & 1 & Pencil & 1 \\
\hline \multicolumn{4}{|l|}{ Active cooling $\left(-20^{\circ} \mathrm{C} / 4^{\circ} \mathrm{C}\right) 2$ packages, $50 \mathrm{~kg}$} \\
\hline Cooling box, CoolFreeze CFX 50, WAECO & 2 & & \\
\hline \multicolumn{4}{|l|}{ Passive transport cooling 2 packages, $40 \mathrm{~kg}$} \\
\hline Mini Vacuum Case, DeltaT & 2 & Cooling elements, DeltaT, $-21^{\circ} \mathrm{C}$ & 10 \\
\hline Cooling elements, DeltaT, $+4^{\circ} \mathrm{C}$ & 10 & Diagnostic reagents according to mission requirements & \\
\hline \multicolumn{4}{|l|}{ Documentation and communication 1 package, $31 \mathrm{~kg}$} \\
\hline Laptop, ruggedised, GETAC V100 & 2 & Inkjet printer cartridges & 3 \\
\hline Mobile document scanner & 1 & Office printer paper, pack & 1 \\
\hline Mobile inkjet printer & 1 & Pen, document-proof & 5 \\
\hline Compact camera, ruggedised, LUMIX DMC-FT5 & 1 & Self-adhesive laboratory labels, numbered consecutively & 500 \\
\hline Satellite phone, Thuraya XT & 1 & USB cable & 2 \\
\hline Laboratory notebook & 2 & USB stick $4 \mathrm{~GB}$, write-protectable & 4 \\
\hline Field laboratory request forms & 500 & First aid kit & 1 \\
\hline \multicolumn{4}{|l|}{ Electricity 2 packages, $50 \mathrm{~kg}$} \\
\hline Power inverter, $12-230 \mathrm{~V}, 1,500 \mathrm{~W}$ & 2 & 5-fold socket, integrated overvoltage protection & 6 \\
\hline Residual-current circuit breaker adapter plugs & 2 & Battery tester & 2 \\
\hline Nitrile examination gloves, large & 200 & Battery connection cables for power inverter & 2 \\
\hline Nitrile examination gloves, medium & 200 & Duct tape & 2 \\
\hline Nitrile examination gloves, small & 200 & Extension cable, $10 \mathrm{~m}$ & 2 \\
\hline Polyethylene construction sheeting, $20 \mathrm{~m}^{2}$ & 2 & International socket adapter plug sets & 2 \\
\hline Working place lights & 4 & & \\
\hline
\end{tabular}


a strict one-way rule for processing of the samples, starting from the sample reception area (Figure 2A), to sample inactivation and nucleic acid isolation (B), ELISA and microscopy (C) and ending in the PCR analysis room (D) to avoid cross-contamination of the samples. In order to reduce the number of separated rooms, the preparation of the PCR master mix is located inside a flexible film isolator designed for material protection. Tables and chairs are not part of the mobile laboratory equipment and have to be acquired locally. They are also covered with construction foil in order to facilitate easy surface decontamination. If electrical power is available locally (from portable generators or power line), power inverters are used as back-up systems and interruption-free power supplies (Figure 2). Each laboratory is equipped with two $1,500 \mathrm{~W}$ power inverters that provide $230 \mathrm{~V} \mathrm{AC}$ power from a car battery.

\section{Logistical support}

Robustness and transportability are key features of our mobile laboratory design. Therefore, all laboratory instruments are selected for size, weight and durability, even under extreme field conditions. The complete laboratory equipment is packed in wheeled, ruggedised, water- and dustproof boxes (Figure 1B). More than 400 items are allocated to 27 boxes (Table 3). Box weights ranging from $20 \mathrm{~kg}$ to a maximum of $31 \mathrm{~kg}$ allow not only their transport as passenger luggage by all international airlines, but also ease handling by the laboratory team during reloading and land transport. Although customs-related import problems are likely to be specific to every deployment, meticulous preparation of foreseeable transport documents (pro-forma invoice, import/export declaration forms, tax exemption certificates and multilingual packing lists) facilitates customs clearance significantly [23].

To guarantee a high degree of flexibility, different sets of equipment are packed for specific deployment missions. If not needed for a certain mission, individual boxes (e.g. for ELISA or microscopy) can be omitted from the deployment without affecting the performance of the other laboratory functions. To ensure the cleanliness of the consumables, such as gloves and reaction tubes, and minimise their space requirement in the boxes, they are packed in daily consumption quantities and vacuum-sealed in multilayer laminated aluminium foil pouches. Consumables and reagents are apportioned to allow continuous operation of a single laboratory for up to 50 days (based on the assumption of handling 25 samples per day) without logistic support. In the current EMLab missions, replacement teams replenish stocks of consumables. Since the active cooling or freezing devices of the laboratory cannot be operated during air transport, vacuum insulated boxes are used to bring temperature-sensitive reagents into the field.

\section{Conclusions}

The latest Ebola outbreak support mission, conducted by the EMLab consortium in several West African countries, has confirmed that rapid turnaround times are critical for surveillance and patient management, and at the same time facilitated unique scientific studies. Besides human diagnostics, the EMLabs provided high-quality laboratory expertise to support research and development activities for the assessment of new Ebola rapid diagnostics, for Ebola treatment trials and for Ebola vaccine research [24-26]. They were also engaged with other partners in operational research to review or confirm public health prevention and control strategies [27]. From March 2014 to October 2015, more than 19,000 samples were tested in the EMLab units. The overall costs for the complete field laboratory equipment described here are ca EUR 150,000, compared with the more than 10 times higher costs of other container- or truck-based solutions (DG DevCo, personal communication, 23 April 2015). Even though some security, safety and health risks are always present in mobile missions, they can be mitigated by careful planning, mission preparation and team training. With these measures, a safe working environment was ensured during more than 1,300 Ebola laboratory mission days in West Africa during the rainy season and the dry season.

The mobile laboratory concept described here is a forward-looking solution for outbreak response in remote areas where emerging infectious diseases might occur. It offers rapidly deployable, state-of-the-art technology for effective field diagnostics capabilities. Our field laboratory concept has served as a blueprint for similar projects and may continue to provide a model for future mobile laboratory projects.

\section{Acknowledgements}

We thank all team members of the military field lab and of the European Mobile Labs for their ideas and feedback in the training and during the lab operations worldwide. Their input influenced many details of the mobile field lab. The Bundeswehr Medical Service has funded the research described herein as part of its Medical Biological Defence Research Programme. The European Mobile Lab project ('Establishment of Mobile Laboratories for Pathogens up to Risk Group 4 in Combination with CBRN Capacity Building in Sub-Saharan Africa') is funded by the European Union, European Union External Actions, project reference IFS/2011/272-372. Opinions, interpretations, conclusions and recommendations are those of the authors and are not necessarily endorsed by any governmental agency, department or other institutions.

\section{Conflict of interest}

The corresponding author is listed as a co-inventor of the foldable glovebox system in a European patent. All other authors indicate no conflicts of interest.

Authors' contributions

RW designed the mobile field-lab concept. EF, BG, PM, LZ, GG, KS and RW developed, assembled and validated the mobile field-lab systems. KS and RW wrote the first draft of the paper. ADC, SG, SI, AOH, PF and LZ critically reviewed the 
first draft of the paper, and their comments were incorporated. All authors read and approved the final manuscript.

\section{References}

1. World Health Organization (WHO). Ebola situation report - 28 October 2015. Geneva: WHO; 2015. Available from: http://apps.who.int/ebola/current-situation/ ebola-situation-report-28-october-2015

2. BaizeS, PannetierD, OestereichL, RiegerT, KoivoguiL, MagassoubaN, et al. Emergence of Zaire Ebola virus disease in Guinea. N Engl J Med. 2014;371(15):1418-25. DOI: 10.1056/ NEJMoa1404505 PMID: 24738640

3. SchieffelinJS, ShafferJG, GobaA, GbakieM, GireSK, ColubriA, et al. Clinical illness and outcomes in patients with Ebola in Sierra Leone. N Engl J Med. 2014;371(22):2092-100. DOI: 10.1056/NEJMoa1411680 PMID: 25353969

4. BoisenML, SchieffelinJS, GobaA, OottamasathienD, JonesAB, Shaffer]G, et al. Multiple circulating infections can mimic the early stages of viral hemorrhagic fevers and possible human exposure to filoviruses in Sierra Leone prior to the 2014 outbreak. Viral Immunol. 2015;28(1):19-31.PMID: 25531344

5. GrollaA, JonesS, KobingerG, SprecherA, GirardG, YaoM, et al. Flexibility of mobile laboratory unit in support of patient management during the 2007 Ebola-Zaire outbreak in the Democratic Republic of Congo. Zoonoses Public Health. 2012;59(Suppl 2):151-7. DOI: 10.1111/j.1863-2378.2012.01477.x PMID: 22958259

6. GrollaA, JonesSM, FernandoL, StrongJE, StröherU, MöllerP, et al. The use of a mobile laboratory unit in support of patient management and epidemiological surveillance during the 2005 Marburg Outbreak in Angola. PLoS Negl Trop Dis. 2011;5(5):e1183. DOI: 10.1371/journal.pntd.0001183 PMID: 21629730

7. InglisTJ. The lab without walls: a deployable approach to tropical infectious diseases.Am J Trop Med Hyg. 2013;88(4):614-8. DOI: 10.4269/ajtmh.12-0704 PMID: 23553225

8. InglisT], MerrittA, MontgomeryJ, Jayasinghel, ThevanesamV, McInnesR. Deployable laboratory response to emergence of melioidosis in central Sri Lanka.J Clin Microbiol. 2008;46(10):3479-81. DOI: 10.1128/JCM.01254-08 PMID: 18716231

9. TownerJS, KhristovaML, SealyTK, VincentMJ, EricksonBR, BawiecDA, et al. Marburgvirus genomics and association with a large hemorrhagic fever outbreak in Angola. J Virol. 2006;80(13):6497-516. DOI: 10.1128/JVI.00069-06 PMID: 16775337

10. North Atlantic Treaty Organization. AEP-66: NATO handbook for sampling and identification of biological, chemical and radiological agents. Brussels: NATO Information Service; 2009.

11. World Health Organization (WHO). Laboratory biosafety manual. 3rd ed. Geneva: WHO; 2004.

12. Kozlovacl, SchmittB. Biosafety principles and practices for the veterinary diagnostic laboratory. Methods Mol Biol. 2015;1247:31-41. DOI: 10.1007/978-1-4939-2004-4_3 PMID: 25399086

13. WhetstoneC, NelsonBJ, WoodsCR. Biosafety in research: oversight and basic principles.Pediatr Infect Dis J. 2010;29(8):763-5. DOI: 10.1097/INF.obo13e3181eaoe31 PMID: 20661104

14. Chosewood LC, Wilson DE, US Centers for Disease Control and Prevention (CDC), US National Institutes of Health. Biosafety in microbiological and biomedical laboratories. 5th ed. Washington DC: US Department of Health and Human Services, Public Health Service, CDC; 2009.

15. SewellDL. Laboratory-associated infections and biosafety.Clin Microbiol Rev. 1995;8(3):389-405.PMID: 7553572

16. BlowJA, DohmDJ, NegleyDL, MoresCN. Virus inactivation by nucleic acid extraction reagents.J Virol Methods. 2004;119(2):195-8. DOI: 10.1016/j.jviromet.2004.03.015 PMID: 15158603

17. MitchellSW, McCormickJB. Physicochemical inactivation of Lassa, Ebola, and Marburg viruses and effect on clinical laboratory analyses.J Clin Microbiol. 1984;20(3):486-9.PMID: 6490832

18. DauphinLA, MoserBD, BowenMD. Evaluation of five commercial nucleic acid extraction kits for their ability to inactivate Bacillus anthracis spores and comparison of DNA yields from spores and spiked environmental samples.J Microbiol Methods. 2009;76(1):30-7. DOI: 10.1016/j.mimet.2008.09.004 PMID: 18824041
19. GrollaA, LuchtA, DickD, StrongJE, FeldmannH. Laboratory diagnosis of Ebola and Marburg hemorrhagic fever.Bull Soc Pathol Exot. 2005;98(3):205-9.PMID: 16267962

20. DIN EN 12469 - Biotechnology - Performance criteria for microbiological safety cabinets: Beuth; 2000.

21. WuSJ, PalS, EkanayakeS, GreenwaldD, LaraS, RaviprakashK, et al. A dry-format field-deployable quantitative reverse transcriptase-polymerase chain reaction assay for diagnosis of dengue infections. Am J Trop Med Hyg. 2008;79(4):505-10. PMID: 18840736

22. McAvinJC, SwansonKI, ChanAS, QuintanaM, ColemanRE. Leishmania detection in sand flies using a field-deployable real-time analytic system.Mil Med. 2012;177(4):460-6. DOI: 10.7205/MILMED-D-11-00206 PMID: 22594139

23. Durgavich J. Customs Clearance Issues Related to the Import of Goods for Public Health Programs. Arlington, VA: United States Agency for International Development; 2009.

24. BaggiFM, TaybiA, KurthA, Van HerpM, Di CaroA, WölfelR, et al. Management of pregnant women infected with Ebola virus in a treatment centre in Guinea, June 2014 Euro Surveill. 2014;19(49):20983. DOI: 10.2807/1560-7917. ES2014.19.49.20983 PMID: 25523968

25. Henao-RestrepoAM, LonginilM, EggerM, DeanNE, EdmundsWJ, CamachoA, et al. Efficacy and effectiveness of an rVSVvectored vaccine expressing Ebola surface glycoprotein: interim results from the Guinea ring vaccination clusterrandomised trial. Lancet. 2015;386(9996):857-66. DOI: 10.1016/So140-6736(15)61117-5 PMID: 26248676

26. MoreauM, SpencerC, GozalbesJG, ColebundersR, LefevreA, GryseelsS, et al. Lactating mothers infected with Ebola virus: EBOV RT-PCR of blood only may be insufficient. Euro Surveill. 2015;20(3):21017. DOI: $10.2807 / 1560-7917 . E S 2015 \cdot 20.3 \cdot 21017$ PMID: 25635320

27. CarrollMW, MatthewsDA, HiscoxJA, ElmoreMJ, PollakisG, RambautA, et al. Temporal and spatial analysis of the 2014-2015 Ebola virus outbreak in West Africa. Nature. 2015;524(7563):97-101. DOI: 10.1038/nature14594 PMID: 26083749 